\title{
Comparando Mecanismos de Redução da Pobreza: Crescimento Econômico ou Programa Bolsa Família?
}

\author{
Gisele De Cássia Gusmão \\ Uyara de Sales Gomide ${ }^{2}$
}

Silvia Harumi Toyoshima ${ }^{3}$

\begin{abstract}
Resumo: Com o objetivo de identificar e comparar a influência do crescimento econômico e do Programa Bolsa Família no combate a pobreza utilizou-se como metodologia de mensuração a Análise Discriminante. Os resultados demonstram que o baixo nível de alfabetização pode ser entendido como característica inerente à maior incidência de pobreza. Em relação ao Programa Bolsa Família, este possui uma relação inversa com incidência de pobreza sugerindo que aumentos no valor dos benefícios transferidos pelo governo promovem a redução da pobreza. Em outras palavras, educação e transferência de renda discriminam os estados brasileiros que estão acima da média (Grupo 1), em termos de nível de pobreza, daqueles que estão abaixo da média (Grupo 2). Por outro lado, o crescimento econômico não explicou a diferença de incidência de pobreza entre os dois grupos.
\end{abstract}

Palavra-chave: Programa Bolsa Família. Crescimento econômico. Pobreza.

JEL: I38.

1 Professora do Departamento de Economia - Universidade Estadual de Montes Claros/Unimontes Mestre em Economia - Universidade Federal de Viçosa/UFV E-mail: giselecgusmao@yahoo.com.br

2 Mestranda em Economia - Universidade Federal do Ceará/CAEN E-mail: uyara.salles@gmail.com

3 Professora Associada do Departamento de Economia - Universidade Federal de Viçosa/UFV

Doutora em Ciência Econômica - Universidade Estadual de Campinas/Unicamp E-mail: htsilvia@ufv.br 


\title{
Comparing Mechanisms for Reduction of Poverty: Economic Growth or Bolsa Família Program?
}

\begin{abstract}
This paper aims to identify and make a comparison of the relationship between economic growth and Bolsa Família Program to combat poverty. To achieve that, a discriminant function was proposed. The results show that low literacy can be understood as characteristic of the higher incidence of poverty. The Bolsa Familia Program has an inverse relationship with poverty incidence suggesting that increases in the value of benefits transferred by the government promote poverty reduction. In other words, education and income transfer programs discriminate brazilian states that are above average (Group 1), in terms of poverty level, those who are below the average (Group 2). Moreover, economic growth did not explain the difference in incidence of poverty between the two groups.
\end{abstract}

Key-words: Bolsa Família Program. Economic growth. Poverty.

JEL: I38.

\section{Introdução}

Um dos graves problemas enfrentados por muitos países em desenvolvimento é a forte concentração de renda e incidência de pobreza. Combater a pobreza é tema recorrente na agenda política desses países e, ganhou maior atenção com a Cúpula do Milênio realizada no ano de 2000, na qual países membros das Nações Unidas assinaram a Declaração do Milênio nela traçados objetivos e metas a serem cumpridos até o ano de 2015. Os Objetivos do Milênio buscam alcançar o desenvolvimento das Nações, dentre eles, o de reduzir a extrema pobreza (PNUD, 2010).

Caracterizar a pobreza é algo extremamente complexo, já que ela pode considerar algum juízo de valor, em termos relativos ou absolutos. Do ponto de vista relativo, a pobreza está relacionada à distribuição de renda e aos fenômenos macroeconômicos. Há um padrão de vida vigente na sociedade, a partir do qual são classificados como pobres aqueles que estão fora desse padrão. Já o enfoque da pobreza absoluta considera uma linha de pobreza que determina o percentual de pessoas que se encontram abaixo dessa linha. A linha que limita os pobres dos não pobres considera um padrão de vida mínimo, em relação aos aspectos nutricionais, de moradia, de vestuários, de lazer, de transporte, dentre outros fatores, a partir do cálculo da renda necessária para adquiri-los (Barros et al. 2000; Crespo \& Gurovitz, 2002; Rocha, 2005; Kageyama \& Hoffman, 2006). 
No Brasil, a pobreza é reflexo da histórica desigualdade de renda. O país se destaca tanto no cenário econômico - como uma das maiores economias do mundo - como na elevada desigualdade de distribuição de renda. Dados contidos na PNUD (2010) apontam que, no Brasil, 8\% da população vive com menos de $\$ 1,25$ (PPP) dólar por dia o que representa 13 milhões de brasileiros. Segundo Sen (2000), pessoas que vivem no Gabão, África do Sul, Namíbia ou Brasil, em termos de PNB per capita, podem ser tituladas como ricas em relação às que vivem em países como Sri Lanka, China ou o Estado de Kerala, na Índia. Contudo, nessas últimas regiões, a população possui expectativas de vida mais elevadas que naquelas. Logo, a extensão do entendimento da pobreza segundo o autor, foge de seu atributo unívoco (ou seja, como insuficiência de renda) passando a considerá-la como uma privação de múltiplas capacidades.

O conceito de capacidades discutido por Sen (2000) combina alternativas de funcionamento de possível realização e também a liberdade do indivíduo poder alcançar estilos de vida diversos. Portanto, a pobreza é vista como um obstáculo que impossibilita o indivíduo de exercer essa liberdade. Quando esta liberdade é observada, o indivíduo possui a capacidade de fazer as escolhas que ele considera importantes para satisfazer as suas necessidades individuais, materiais e não materiais.

Com isso, na tentativa de integrar os mais pobres ao processo de crescimento, países em desenvolvimento buscam promover políticas sociais e estruturais que sejam pró-pobre.

A definição de crescimento pró-pobre não é unívoca. Segundo Ravallion e Chen (2003), há duas definições: $1^{\mathrm{a}}$ ) crescimento pró-pobre significa uma queda na pobreza maior do que se teria caso todas as rendas tivessem a mesma taxa de crescimento; e, $2^{\mathrm{a}}$ ) crescimento pró-pobre é crescimento que reduz pobreza. Diante disso, o crescimento econômico ganha importância quando inclusivo e favorável às camadas mais pobres. A não ocorrência desta condição pode não permitir que um país, como o Brasil, com índice de desigualdade elevado, experimente um crescimento pró-pobre significativo, evitando assim, consequências que podem repercutir num ciclo perverso de pobreza e encadeador de outros problemas econômicos e sociais.

A elasticidade da pobreza em relação ao crescimento mede a mudança na pobreza absoluta dado $1 \%$ de mudança no crescimento. Se uma parcela significativa da população estiver perto da linha da pobreza, um pequeno aumento no crescimento levará essa proporção da população para além da linha, o que mostra que a pobreza é sensível ao crescimento.

Salvatto et al. (2007) buscaram verificar a ocorrência de crescimento própobre no Brasil, e conclúram que tal fenômeno ocorreu em quase $25 \%$ dos 5.506 municípios brasileiros (excluindo o Distrito Federal). Os Estados do 
Ceará e de Pernambuco apresentaram os piores desempenhos nesse sentido, respectivamente, em 3,26\% e 3,78\% dos municípios. No entanto, sabe-se que os estados do Nordeste são os que registram maior número de população que vive na zona rural, o que pode influenciar na elasticidade pobreza-crescimento nesta região já que o crescimento econômico tende a gerar mais benefícios para a população que vive na zona urbana.

No Brasil, caso o crescimento fosse de apenas $2 \%$, a redução da pobreza seria de cinco pontos percentuais, ao passo que um crescimento contínuo de $5 \%$ a.a. levaria, em uma década, a uma redução de 13 pontos percentuais no grau de pobreza (Barros et al.1997). Segundo Kakwani et al. (2006), a renda per capita brasileira recuou no período de 2000 a 2004; porém, a pobreza e a desigualdade diminuiu. A explicação para os autores é que, enquanto o mercado de trabalho foi adversamente afetado, rendas provenientes de seguros sociais e outras transferências governamentais tiveram uma função crucial na contenção das consequências dos grandes choques observados, especialmente entre os segmentos mais pobres da sociedade brasileira.

Para Rocha (2005:21)

"embora crescimento econômico não seja uma panacéia, garantir um ritmo sustentado da expansão da renda nacional tornaria, sem dúvida, mais fácil estabelecer os mecanismos institucionais necessários ao combate à pobreza, já que a persistência de pobreza absoluta no Brasil está vinculada à elevada desigualdade de renda e a uma estrutura inadequada do gasto social”.

Nesta perspectiva, ganham destaque os programas de transferência de renda que objetivam reforçar o capital humano não somente no sentido de educar a mão de obra, como também melhorar as condições de saúde, nutrição e alimentação do grupo familiar beneficiado que se encontra em condições de pobreza que, para Villatoro (2005), significa maior vulnerabilidade, uma vez que os pobres têm pouco acesso aos instrumentos adequados para administrar os riscos e lidar com crises.

Diversas são as experiências de programas de transferência de renda nos países em desenvolvimento. Os trabalhos de Mallucio \& Flores (2005), Brierè e Rawlings (2006), Hoddinotti et al. (2000), Villatoro (2005) e Coady \& Parker (2002) apresentam resultados alcançados nas áreas de educação, saúde e nutrição nos países em desenvolvimento com a implantação de programas de transferência de renda como o programa Familias en Acción, na Colômbia; a Rede de Proteção Social, na Nicarágua; o programa Oportunidades, no México; o Programa de Asignación Familiar (PRAF) em Honduras, o Programa Bolsa Família, no Brasil, dentre outros.

Os resultados indicaram que os programas de transferência de renda visam melhorar o capital humano das crianças e dos jovens, a fim de quebrar o ciclo intergeracional da pobreza, incentivando as famílias a investirem em 
educação, nutrição e saúde. Estes programas decorrem de uma abordagem que prioriza lidar com as causas da pobreza e, portanto, apontam que, a longo prazo, a tendência é reduzir a pobreza. Por servirem essencialmente para atender a população que se encontra em situação de pobreza estrutural, aperfeiçoar o capital humano significa reduzir os riscos futuros.

O que a experiência na implementação desses programas tem revelado, segundo Villatoro (2005) é que o custo para mantê-los não é incompatível com países pobres, pois representam uma pequena parcela dos orçamentos nacionais.

No Brasil, ganha destaque como política social de renda mínima para melhorar a distribuição de renda no país e reduzir a incidência de pobreza, $o$ Programa Bolsa Família (PBF) ${ }^{1}$. Considerado um dos maiores programas de transferência de renda do mundo, o Bolsa Família propõe combater a pobreza e a fome; promover o acesso aos serviços públicos de saúde e educação; promover a segurança alimentar e nutricional dos beneficiados e, assim, amenizar as condições de vida precárias assistidas pelas famílias classificadas como pobres e extremamente pobres, seu público alvo (MDS, 2010).

Após a criação do Ministério do Desenvolvimento Social e Combate à Fome (MDS) em 2004, iniciou-se um processo de "migração" dos beneficiários de antigos programas de transferência de renda (Auxílio Gás, Bolsa Escola, Bolsa Alimentação e Cartão Alimentação) para um programa que consolidasse todas estas ações e atendesse a família como um núcleo formado por membros com diferentes necessidades, resultando na criação do PBF.

Criado pela Lei $\mathrm{n}^{\circ}$ 10.836, de 9 de janeiro de 2004, o PBF resgata uma das características dos programas anteriores similares, vale dizer, o benefício variável por número de crianças da família, no total de até três. No entanto, inova quando elege a família ${ }^{2}$ como beneficiária, e não cada um de seus membros isoladamente, como nos casos anteriores - Bolsa Escola e Bolsa Alimentação. Inova também ao não estipular quotas de número de bolsas para cada município (Santana, 2007).

A seleção dos beneficiários do Programa Bolsa Família é conduzida nos municípios empregando um método de comprovação de insuficiência de renda auto-declarada (não certificada). O alvo do programa são famílias extremamente pobres e famílias pobres que têm gestantes, crianças com até 15 anos de idade e adolescentes de 16 e 17 anos. O Bolsa Família é hoje o maior programa federal de transferência de renda do país atingindo, no ano

\footnotetext{
1 No ano de 2007, a cidade de Nova York divulgou o programa de transferência condicional de renda "Opportunity NYC", espelhado no programa brasileiro e no programa mexicano, Opportunidades. Rara ocasião em que um país em desenvolvimento adota e aprende com experiências de programas modelados nos países em desenvolvimento (PNUD, 2010).

2 Família pode ser definida como a unidade nuclear, eventualmente ampliada por outros indivíduos que com ela possuam laços de parentesco ou de afinidade, que forme um grupo doméstico, vivendo sob o mesmo teto e que se mantém pela contribuição de seus membros (Brasil, 2004).
} 
de 2010, mais de 12 milhões de famílias com renda familiar por pessoa de até $\mathrm{R}$ \$ 140,00. O valor do benefício transferido às famílias pode variar entre $\mathrm{R} \$$ 22,00 e R\$200,00, a depender do número e da idade dos filhos e da renda per capita (MDS, 2010).

O Bolsa Família além de contribuir para o incremento da renda familiar incentiva o acesso aos serviços públicos de saúde e educação por meio das condicionalidades - nas áreas da saúde, educação e assistência social - que é uma forma de assegurar o compromisso dos beneficiários com o Programa. O não cumprimento das condicionalidades leva às advertências, bloqueando o benefício, e até ao desligamento da família. Caso a família advertida não concorde com a notificação a ela encaminhada, a mesma pode recorrer da advertência por meio de um formulário disponível com o gestor municipal. Se for comprovado que as famílias não cumpriram as condicionalidades porque a prefeitura não ofereceu os serviços necessários para o seu cumprimento, não será aplicada à família nenhuma sanção.

O papel das condicionalidades mais do que garantir ao governo o comprometimento das famílias frente ao investimento público, reforça a participação efetiva da população pobre na sociedade, ao possibilitar o acesso e exercício de seus direitos nas áreas de educação, saúde e assistência social. Reforça também, o compromisso do gestor local em oferecer serviços nessas áreas, uma vez que as famílias necessitam dos mesmos para cumprir as condicionalidades. A utilização desses serviços é uma forma do programa atingir a sua meta que é de retirar as famílias da situação de vulnerabilidade social. O MDS considera as famílias mais vulneráveis socialmente, aquelas que deixam de cumprir as condicionalidades.

Em síntese, o Bolsa Família pretende aliar dois objetivos centrais: primeiro, combater a pobreza intergeracional, incentivando o acesso à educação a fim de melhorar, no longo prazo, o capital humano e; segundo, garantir renda para a satisfação das necessidades básicas da família minorando as privações do grupo familiar, no curto prazo. A garantia de proteção social impede que os indivíduos que vivem em condições vulnerabilidade sejam adversamente afetados e levados a condições de extrema carência devido às mudanças intrínsecas a toda economia em processo de desenvolvimento, Portanto, esses programas são importantes para remover o que impede o indivíduo de exercer suas capacidades.

Isso posto, o presente estudo tem como objetivo identificar e comparar a relação do crescimento econômico e do Programa Bolsa Família no combate à pobreza no período de 2004 a 2007, para o Brasil, utilizando um modelo de Análise Discriminante. Além desta Introdução, o estudo segue com as seguintes seções: na segunda parte, apresenta-se a metodologia; na terceira parte, encontram-se os resultados e discussão e, por fim, as considerações finais. 


\section{Metodologia}

Com o intuito de identificar e comparar a influência do crescimento econômico e do Programa Bolsa Família no combate à pobreza foi utilizado um modelo de Análise Discriminante ${ }^{3}$. O objetivo da Análise Discriminante (AD) - uma técnica estatística multivariada - é verificar as diferenças existentes entre grupos definidos, a priori, a partir de uma série de variáveis independentes. Para tanto, foram definidos os seguintes grupos: o Grupo 1 refere-se aos estados brasileiros com incidência de pobreza abaixo da média nacional e, o Grupo 2 àqueles com nível de pobreza acima da média. Essa divisão se deu apenas pela comparação entre os valores do índice de pobreza e as médias para cada ano de todos os estados brasileiros. Esperou-se que a maioria dos estados, principalmente os pertencentes às Regiões Norte e Nordeste, fossem classificados no Grupo 2.

A representação das diferenças entre os grupos é descrita a partir de uma ou mais funções discriminantes que "é composta pelas variáveis independentes que representam as características do elemento, que são ponderadas pelo nível de sua importância ou impacto que causam no resultado ou variável dependente" (Mário, 2007: 236).

A função linear discriminante é representada da seguinte forma:

$$
Z=a+b_{1} X_{1}+b_{2} X_{2}+\ldots+b_{n} X_{n},
$$

Onde $Z$ é o score (pontuação) discriminante; $a$ é o intercepto da função quando todo $X_{i}=0 ; b_{n}$ é o coeficiente discriminante ou a capacidade que cada variável independente tem em discriminar (o peso de cada uma na função); e $X_{n}$ são os valores das variáveis independentes. Em suma, a função discriminante possibilita entender como um conjunto de variáveis influencia simultaneamente no comportamento de um elemento.

Desta esquação, calcula-se um score discriminante para cada caso analisado. Para analisar os scores discriminantes médios para todos os casos de um grupo particular, chega-se à média do grupo, que é referida como centróide.

Os centróides tem a função de realizar uma melhor localização de qualquer caso de um grupo particular definido a priori e a comparação desses centróides objetiva identificar como estão separados a partir das variáveis independentes selecionadas. Deste modo, quanto mais distante os centróides estiverem, tanto mais as variáveis selecionadas estarão diferenciando os grupos. $O$ teste para significância estatística da função discriminante é medida pela distância entre os centróides dos grupos como mostra as Figuras 1 e 2. Se, por exemplo, o número de grupos que se quer separar seja dois, os scores discriminantes

3 Metodologia adotada baseia-se em Hair et al., 2005. 
calculados para cada observação serão distribuídos em duas normais (esse é o efeito desejado), como mostram as Figuras 1 e 2.

\section{Figura 1. DISTRIBUIÇÃO DOS SCORES DISCRIMINANTES SIGNIFICATIVA.}

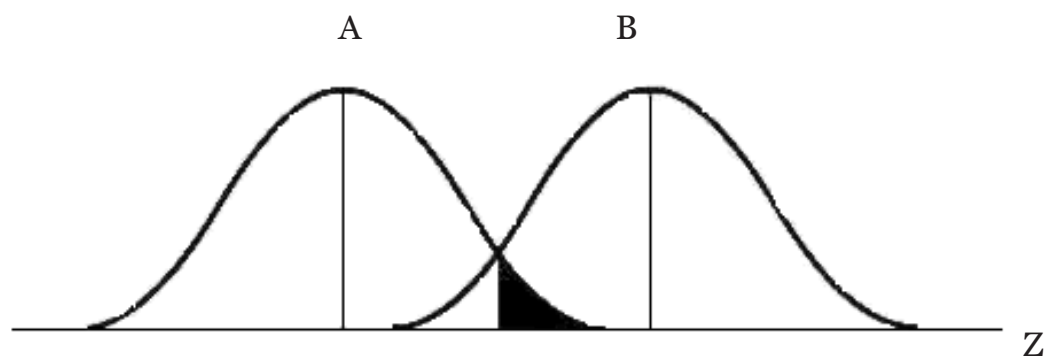

Função Discriminante

Fonte: Elaborado pelos autores

Figura 2. DISTRIBUIÇÃO DOS SCORES DISCRIMINANTES NÃOSIGNIFICATIVA.

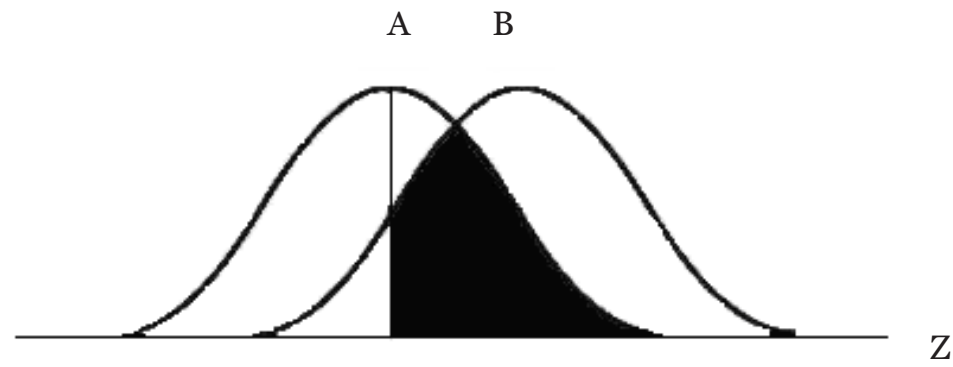

Função Discriminante

Fonte: Elaborado pelos autores

Caso tais distribuições se assemelharem à figura 1, as variáveis estarão diferenciando bem os grupos. Se, por outro lado, serem mais semelhantes 
à Figura 2, então a discriminação é pobre, o que significa que as variáveis selecionadas não são as mais adequadas para a separação das observações em dois grupos. Quanto maior a sobreposição dos grupos A e B, pior é a discriminação. A área hachurada representa, em ambas as figuras, a probabilidade de casos mal classificados entre A e B. Esse procedimento envolve o exame das funções discriminantes para determinar a importância proporcional ou relativa de todas as variáveis independentes na discriminação entre os grupos por meio de três métodos: pesos discriminantes padronizados, cargas discriminantes (estruturas correlacionadas) e $\mathrm{F}$ valores parciais.

A seleção das variáveis com maior poder discriminatório foi estabelecida por meio do método stepwise. Trata-se de um processo de estimação de funções discriminantes no qual variáveis independentes entram sequencialmente de acordo com o poder discriminatório que elas apresentam à previsão de pertinência no grupo (Hair et al. 2005). Analisa-se o sinal e a magnitude do peso discriminante das variáveis independentes. Variáveis independentes com pesos relativamente grandes contribuem muito mais para o poder discriminante da função do que variáveis com valores pequenos. Variáveis com pesos muito pequenos podem ser colocadas para fora da análise, pois podem apresentar multicolinearidade. $\mathrm{O}$ sinal apenas mostra se a variável apresenta uma contribuição direta ou inversa à variável independente.

Empregou-se uma variação do teste F, Lambda Wilk's (ou, estatística U), a fim de se analisar o modelo discriminante como um todo. Este representa a proporção da variabilidade total que não é explicada pelas diferenças entre os grupos. Testa, na realidade, a igualdade dos centróides ${ }^{4}$ dos grupos. Valores próximos a zero são os desejados, pois indicam forte diferença entre as médias (Corrar et al. 2009).

Sendo assim, testou-se a seguinte função discriminante:

$$
P_{i j}=\alpha+\beta_{1} P I B_{i j}+\beta_{2} B F_{i j}+\beta_{3} G I N I_{i j}+\beta_{4} A N A L F_{i j}
$$

Onde $i$ corresponde ao estado e $j$ ao ano, em estudo. $P_{i j}$ corresponde ao índice de pobreza; $\alpha$ é o intercepto; $\beta$ é o peso discriminante para a variável independente, ou seja, a capacidade que cada variável independente possui em discriminar; $P I B_{i j}$ corresponde ao PIB per capita por estado; $B F_{i j}$ é o valor total do benefício pelo número de pobres para cada estado; $G I N I_{i j}$ é a medida de concentração de renda existente em cada estado; e, $A N A L F_{i j}$, proxi da educação, que representa o percentual de pessoas de 15 ou mais anos de idade que não sabem ler nem escrever um bilhete simples.

Para este estudo foram utilizadas informações disponíveis no banco de dados do Ipeadata para os anos de 2004 a 2007.

4 Valor Médio para os escores $\mathrm{Z}$ discriminantes de todos os objetos, em uma dada categoria ou grupo. 


\section{Resultados}

A Tabela 1 apresenta os valores dos coeficientes padronizados das funções discriminantes geradas pelo modelo. As funções discriminantes geradas a partir do método stepwise incluíram as variáveis ANALF e BF indicando que estas duas variáveis são significativas na diferenciação entre os grupos. As variáveis PIB e Índice de Gini não foram significativas para discriminar os grupos de estados mais e menos pobres no período analisado. Tal evidência sugere que seria necessário uma maior taxa de crescimento econômico aliada a uma menor concentração de renda para gerar maior impacto sobre a redução da pobreza e inclusão dos pobres. Vale ressaltar que somente no ano de 2005, a variável PIB foi inserida. Os sentidos dos coeficientes encontrados para as variáveis ANALF e BF se invertem no ano de 2005. Desta forma, os resultados atípicos de 2005 e os dados de analfabetismo indicam possíveis problemas com essa variável nesse ano específico, uma vez que, para alguns estados, este aumenta de 2004 para 2005, para se reduzir em seguida, o que não é provável que ocorra para esse tipo de indicador. Nesse sentido, desconsiderou-se a análise para 2005.

TABELA 1. COEFICIENTES PADRONIZADOS DAS FUNÇÕES DISCRIMINANTES GERADAS

\begin{tabular}{l|c|c|c}
\hline \multicolumn{1}{c|}{ Variáveis } & 2004 & 2006 & 2007 \\
\hline BF & $-0,727$ & $-0,843$ & $-0,821$ \\
ANALF & 1,375 & 1,187 & 1,107 \\
PIB & ----- & ----- & ---- \\
\hline
\end{tabular}

Fonte: Elaboração própria os autores.

APara todos os anos analisados, os coeficientes encontrados para a variável ANALF apresentaram uma relação positiva com o índice de pobreza, de forma que, um baixo nível de alfabetização pode ser entendido como característica inerente à maior incidência de pobreza. Já os valores calculados para os coeficientes da variável $\mathrm{BF}$, possuem uma relação inversa com o índice de pobreza, o que sugere que aumentos no valor dos benefícios transferidos pelo governo promovem uma redução na pobreza, ou seja, geram impacto imediato.

Para se avaliar a significância estatística do poder discriminatório das funções discriminantes a estatística U foi utilizada. A Tabela 2 expõe os valores encontrados para o Lambda Wilk's e o nível de significância da função como um todo. 
TABELA 2. VALORES OBTIDOS PARA O LAMBDA WILK'S

\begin{tabular}{l|c|c}
\hline \multicolumn{1}{c|}{ Ano } & Lambda Wilk's & Nível de significância \\
\hline $\mathbf{2 0 0 4}$ & 0,356 & 0,000 \\
$\mathbf{2 0 0 6}$ & 0,330 & 0,000 \\
$\mathbf{2 0 0 7}$ & 0,276 & 0,000 \\
\hline
\end{tabular}

Fonte: Elaboração própria dos autores.

Para os dois testes, quanto mais próximos de zero forem seus valores maior é a capacidade da função em discriminar os elementos entre os grupos. Os valores obtidos para o teste Lambda Wilk's e o nível de significância são os desejáveis. Finalmente, é necessário verificar a proporção das variáveis que foram corretamente classificadas nos grupos (Tabela 3). Além dos resultados gerais, podem-se examinar as observações individuais no que se refere à precisão preditiva e identificar especificamente os casos mal classificados (Anexo).

TABELA 3. PROPORÇÃO DAS VARIÁVEIS CORRETAMENTE CLASSIFICADAS

\begin{tabular}{c|c}
\hline Ano & \% \\
\hline $\mathbf{2 0 0 4}$ & 88,9 \\
$\mathbf{2 0 0 6}$ & 88,9 \\
$\mathbf{2 0 0 7}$ & 96,3
\end{tabular}

Fonte: Resultado da perquisa.

Observa-se que a maioria das variáveis foi corretamente classificada entre os grupos ao longo dos anos, ou seja, a função discriminante classificou as variáveis e explicou a variância de maneira satisfatória. $\mathrm{O}$ ano que apresenta uma melhor predição foi o de 2007 mostrando a elevação nos valores dos resultados.

Os Estados do Amazonas e de Roraima foram os que mais apresentaram erros de acordo com a classificação prevista nos grupos segundo o modelo (Anexo). Em concordância com os dados coletados, os Estados do Amazonas e Roraima possuem incidência de pobreza acima da média, mas o modelo os enquadra no Grupo 1.

A partir dos resultados obtidos pode-se concluir que as funções discriminantes geradas pelo modelo são significantes e que as variáveis que mais contribuem na discriminação entre os grupos são: o valor total do benefício pelo número de pobres para cada estado e o percentual de pessoas de 15 ou mais anos de idade que não sabem ler nem escrever um bilhete simples. 


\section{Considerações finais}

Um dos graves problemas enfrentados por muitos países em desenvolvimento é a grande concentração de renda e forte incidência de pobreza. Desta forma, na tentativa de integrar os mais pobres no processo de crescimento, esses países buscam promover políticas sociais e estruturais que sejam pró-pobre. Nesta perspectiva, o objetivo central deste artigo foi identificar e comparar a influência do crescimento econômico e do Programa Bolsa Família no combate à pobreza por meio de um modelo de Análise Discriminante para o período de 2004 a 2007. Para isso, dois grupos fizeram parte da análise: um grupo de estados que apresentava um índice de pobreza acima da média nacional e, um formado por aqueles abaixo desta média. Os resultados sugerem que o valor do benefício do Programa Bolsa Família, assim como o nível educacional, são significativos para discriminar os dois grupos. Por outro lado, o crescimento econômico e a desigualdade de renda não foram significativos para explicar a incidência de pobreza. Isso não exclui a relevância desses indicadores sobre a redução da pobreza e inclusão dos pobres. No entanto, as outras duas variáveis tiveram maior peso para o alcance dessas metas.

\section{Referências}

BARROS, R. P. de; MENDONÇA, R. (1997). "O impacto do crescimento econômico e de reduções no grau de desigualdade sobre a pobreza”. Texto para discussão $n^{\circ} 528$, IPEA.

BARROS, R. P. de; HENRIQUES, R.; MENDONÇA, R. (2000). "Desigualdade e pobreza no Brasil: retrato de uma estabilidade inaceitável". RBCS Vol. $15 n^{\circ} 42$.

BRASIL. Lei no ${ }^{0}$ 10.386, de 9 de janeiro de 2004.

BRIÈRE, B. de L.; RAWLINGS, L.B. (2006). "Examining Conditional Cash Transfer Programs: A Role for Increased Social Inclusion?” The World Bank. Social Protection Discussion Paper No. 0603, Washington, DC.

CORRAR, L. J.; PAULO, E.; DIAS FILHO, J. M. (2009). Análise Multivariada. São Paulo: Atlas.

COADY, D.P.; PARKER, S.W. (2002). "Cost-effectiveness Analysis of Demandand Supply side Education Interventions: the Case of PROGRESA in Mexico". Discussion Paper, No. 127. International Food Policy Research Institute (IFPRI).

CRESPO, A.P.A; GUROVITZ, E. (2002). A pobreza como um fenômeno multidimensional. RAE-eletrônica, Editora: Fundação Getulio Vargas. Volume 1, Número 2. 
HAIR, J.F.J., ANDERSON, R.E., TATHAM, R.L.; BLACK, W.C. (2005). Análise multivariada de dados. Quinta edição. Porto Alegre: Bookman, 593 p.

HODDINOTT, J.; SKOUFIAS, E.; WASHBURN, R. (2000). "El impacto de Progresa sobre el consumo: informe final”. Informe presentado a PROGRESA. Washington, D.C.: Instituto Internacional de Investigaciones sobre Políticas Alimentarias.

KAGEYAMA, A.; HOFFMANN, R. (2006). "Pobreza no Brasil: uma perspectiva multidimensional”. Economia e Sociedade, Campinas, v. 15, n. 1 (26), p. 79-112.

KAKWANI, N; NERI, M.; SON, H.H. (2006). "Pro-Poor Growth and Social Programmes in Brazil”. Ensaios Econômicos n.639, Rio de Janeiro: FGV.

RAVALLION, M., CHEN, S. (2003). "Measuring pro-poor growth". Development Research Group, World Bank.

ROCHA, S. (2005). "Pobreza no Brasil: O que mudou nos últimos 30 anos?” In: J.P.R. Velloso; R.C. Albuquerque. (Org.). Cinco Décadas de Questão Social e os Grandes Desafios do Crescimento Sustentado. Rio de Janeiro: José Olympio Editora, v., p. 117-220.

SALVATO, M.A; ARAÚJO JUNIOR, A. F. de; MESQUITA, L. A (2007). "Crescimento pró-pobre no Brasil: uma avaliação empírica da década de 1990”. Working paper, 43, Belo Horizonte: IBMEC/MG.

MALUCCIO, J.A.; FLORES, R. (2005). "Impact Evaluation of a conditional cash transfer program: the Nicaraguan Red De Protección Social”. Research Report No. 141. Washington, D.C.: International Food Policy Research Institute.

MARIO, Poueri do Carmo. (2007). “Análise Discriminante”. In: Análise Multivariada para os cursos de Administração, Ciências Contábeis e Economia. Organizadores: Luiz J. Corrar, Edilson Paulo, José Maria Dias Filho. São Paulo: Atlas.

MDS - MINISTÉRIO DO DESENVOLVIMENTO SOCIAL E COMBATE À FOME. URL [on-line]: <http://www.mds.gov.br>.

MINGOTI, S. A. (2005). Análise de dados através de métodos de estatística multivariada: uma abordagem aplicada. Belo Horizonte: Editora, UFMG. 297p.

PNUD - PROGRAMA DAS NAÇÕES UNIDAS PARA O DESENVOLVIMENTO. URL [on-line]: <http://www.pnud.org.br>.

SANTANA, J. A. (2007). A evolução dos programas de transferência de renda e o Programa Bolsa Família. Trabalho apresentado no Seminário População, Pobreza e Desigualdade, Belo Horizonte.

SEN, A.K. (2000). Desenvolvimento como liberdade. São Paulo: Companhia de letras. 
GUSMÃO, G. C.; GOMIDE, U. S.; TOYOSHIMA, S. H. Comparando Mecanismos da Redução...

VILlATORO, P. “Conditional Cash Transfer Programs: Experiences from Latin America”. CEPAL Review 86: 83-96. 2005.

\section{Anexo}

Previsões de grupo para os casos individuais na análise discriminante dos dois grupos

\begin{tabular}{|c|c|c|c|c|c|c|c|c|}
\hline Estados & $\begin{array}{c}\text { Grupo } \\
\text { Atual } \\
(2004)\end{array}$ & $\begin{array}{c}\text { Classificação } \\
\text { pelo } \\
\text { Modelo } \\
\text { (2004) }\end{array}$ & $\begin{array}{c}\text { Grupo } \\
\text { Atual } \\
(2005)\end{array}$ & $\begin{array}{c}\text { Classificação } \\
\text { pelo } \\
\text { Modelo } \\
(2005)\end{array}$ & $\begin{array}{c}\text { Grupo } \\
\text { Atual } \\
(2006)\end{array}$ & $\begin{array}{c}\text { Classificação } \\
\text { pelo } \\
\text { Modelo } \\
\text { (2006) }\end{array}$ & $\begin{array}{c}\text { Grupo } \\
\text { Atual } \\
(2007)\end{array}$ & $\begin{array}{c}\text { Classificação } \\
\text { pelo } \\
\text { Modelo } \\
(2007)\end{array}$ \\
\hline $\mathrm{AC}$ & 2 & 2 & 2 & 2 & 2 & 2 & 2 & 2 \\
\hline $\mathrm{AL}$ & 2 & 2 & 2 & 2 & 2 & 2 & 2 & 2 \\
\hline $\mathrm{AM}$ & 2 & $1^{* *}$ & 2 & $1^{* *}$ & 2 & $1^{* *}$ & 2 & 2 \\
\hline $\mathrm{AP}$ & 2 & $1^{* *}$ & 2 & 2 & 2 & 2 & 2 & 2 \\
\hline $\mathrm{BA}$ & 2 & 2 & 2 & 2 & 2 & 2 & 2 & 2 \\
\hline $\mathrm{CE}$ & 2 & 2 & 2 & 2 & 2 & 2 & 2 & 2 \\
\hline $\mathrm{DF}$ & 1 & 1 & 1 & 1 & 1 & 1 & 1 & 1 \\
\hline ES & 1 & 1 & 1 & 1 & 1 & 1 & 1 & 1 \\
\hline GO & 1 & 1 & 1 & $2^{* *}$ & 1 & 1 & 1 & 1 \\
\hline MA & 2 & 2 & 2 & 2 & 2 & 2 & 2 & 2 \\
\hline $\mathrm{MG}$ & 1 & 1 & 1 & 1 & 1 & 1 & 1 & 1 \\
\hline MS & 1 & 1 & 1 & 1 & 1 & 1 & 1 & 1 \\
\hline MT & 1 & 1 & 1 & 1 & 1 & 1 & 1 & 1 \\
\hline PA & 2 & 2 & 2 & 2 & 2 & 2 & 2 & 2 \\
\hline PB & 2 & 2 & 2 & 2 & 2 & 2 & 2 & 2 \\
\hline PE & 2 & 2 & 2 & 2 & 2 & 2 & 2 & 2 \\
\hline $\mathrm{PI}$ & 2 & 2 & 2 & 2 & 2 & 2 & 2 & 2 \\
\hline PR & 1 & 1 & 1 & 1 & 1 & 1 & 1 & 1 \\
\hline RJ & 1 & 1 & 1 & 1 & 1 & 1 & 1 & 1 \\
\hline $\mathrm{RN}$ & 2 & 2 & 2 & 2 & 2 & 2 & 2 & 2 \\
\hline $\mathrm{RO}$ & 1 & 1 & 2 & 2 & 1 & $2^{* *}$ & 1 & 1 \\
\hline $\mathrm{RR}$ & 2 & $1^{* *}$ & 2 & 2 & 2 & $1^{* *}$ & 2 & $1^{* *}$ \\
\hline RS & 1 & 1 & 1 & 1 & 1 & 1 & 1 & 1 \\
\hline
\end{tabular}


GUSMÃO, G. C.; GOMIDE, U. S.; TOYOSHIMA, S. H. Comparando Mecanismos da Redução...

\begin{tabular}{l|c|c|c|c|c|c|c|c}
\hline Estados & $\begin{array}{c}\text { Grupo } \\
\text { Atual } \\
(2004)\end{array}$ & $\begin{array}{c}\text { Classificação } \\
\text { pelo } \\
\text { Modelo } \\
(2004)\end{array}$ & $\begin{array}{c}\text { Grupo } \\
\text { Atual } \\
(2005)\end{array}$ & $\begin{array}{c}\text { Classificação } \\
\text { pelo } \\
\text { Modelo } \\
(2005)\end{array}$ & $\begin{array}{c}\text { Grupo } \\
\text { Atual } \\
(2006)\end{array}$ & $\begin{array}{c}\text { Classificação } \\
\text { pelo } \\
\text { Modelo } \\
(2006)\end{array}$ & $\begin{array}{c}\text { Grupo } \\
\text { Atual } \\
(2007)\end{array}$ & $\begin{array}{c}\text { Classificação } \\
\text { pelo } \\
\text { Modelo } \\
(2007)\end{array}$ \\
\hline SC & 1 & 1 & 1 & 1 & 1 & 1 & 1 & 1 \\
SE & 2 & 2 & 2 & 2 & 2 & 2 & 2 & 2 \\
SP & 1 & 1 & 1 & 1 & 1 & 1 & 1 & 1 \\
TO & 2 & 2 & 2 & 2 & 2 & 2 & 2 & 2 \\
\hline
\end{tabular}

Fonte: Elaboração própria dos autores

** Erros

Recebido em: 19 de maio de 2012 Aceito em: 2 de maio de 2013 
